\title{
Novel Metrics for Validation of PIV and CFD in IC Engines
}

\section{Blane Scott, Christopher Willman and Richard Stone}

University of Oxford

\author{
David Richardson \\ Jaguar Land Rover Limited
}

\begin{abstract}
In-cylinder flow motion has a significant effect on mixture preparation and combustion. Therefore, it is vital that CFD engine simulations are capable of accurately predicting the in-cylinder velocity fields. Highspeed planar Particle Image Velocimetry (PIV) experiments have been performed on a single-cylinder GDI optical engine in order to validate CFD simulations for a range of engine conditions. Novel metrics have been developed to quantify the differences between experimental and simulated velocity fields in both alignment and magnitude. The Weighted Relevance Index (WRI) is a variation of the standard Relevance Index that accounts for the local velocity magnitudes to provide a robust comparison of the alignment between two vector fields. Similarly, the Weighted Magnitude Index (WMI) quantifies the differences in the local magnitudes of the two velocity fields. The WRI and WMI are normalised and combined to produce a combined metric, the Combined Magnitude and Relevance Index (CMRI), that quantifies the differences between two flow fields in both magnitude and alignment simultaneously.
\end{abstract}

PIV measurements were made every $5^{\circ} \mathrm{ca}$ in the central tumble plane during the induction and compression strokes. The WRI, WMI and CMRI metrics are used to validate numerical simulations of the motored in-cylinder flow measured with PIV for a range of valve lift profiles and engine speeds.

\section{Introduction}

Internal Combustion Engines (ICE) have remained the most popular form of powertrain for ground transportation for the last century. Due to the high energy density of liquid hydrocarbon fuels and extensive existing infrastructure, coupled with the limitations of automotive battery systems, it is likely that the ICE will remain popular in the coming decades [1][2]. Therefore, it is vital that the development of ICEs continues, with the main drivers being the reduction of fuel consumption and exhaust emissions.

To reduce the time and cost of the development of new combustion system designs, Computational Fluid Dynamics (CFD) simulations are now a standard tool. Numerical simulations reduce the number of prototypes that must be built. Furthermore, in the drive for increased efficiency and reduced emissions, modern ICEs use a combination of advanced fuel injection systems and valve strategies to optimize mixture preparation and combustion. The use of such complex systems has led to a large increase in the number of parameters that must be optimized over the whole engine load map. CFD can be used to reduce the amount of engine testing and time taken for calibration.

Page 1 of 14
However, the use of CFD in the development of production engines requires accurate simulation of the physical in-cylinder processes. Therefore, experimental validation is key to the development of the underlying models that are necessary for accurate CFD simulations. For this reason, engines with optical access are a useful tool for providing insights into the in-cylinder processes that would be unavailable with conventional engine testing [3][4].

One of the most important processes that has a significant effect on mixture preparation and combustion is in-cylinder flow motion [5]. It is essential that CFD simulations can accurately predict the development of the flow field during the induction and compression strokes. Particle Image Velocimetry (PIV) is now the standard technique for measuring in-cylinder flow fields and is a useful source of validation data for CFD simulations [6].

\section{Methods of Comparing Vector Fields}

A range of methods have been used to compare simulated flow fields to experimental data in order to assess the effectiveness of the models in capturing the physical processes. A common, qualitative method of comparing flow fields is to plot them side-by-side and search for obvious differences between the flows [7]. Although this is a quick and simple way of spotting discrepancies between simulation and experiment, it is not practical for comparing large datasets. Therefore, quantitative metrics are preferred for robust comparisons.

The investigation of characteristic flow features has been a useful way of providing quantitative comparisons between flow fields, both simulated and experimental. Ameen et al. [8] compared both the mean and RMS velocity magnitudes along the axis of the intake jet to investigate the performance of grid resolution of LES simulations in matching PIV experiments. As well as the intake jet, Yang et al. [9][10] identified the location of vortex centres and the direction of the flow in different regions of the cylinder as important criteria when comparing RANS and LES simulations with PIV experiments. In a similar way, Pera et al. [11] compared the radial and vertical velocity components along two horizontal lines at different in-cylinder locations in order to quantify the difference between LES simulations and PIV experiments.

In contrast to selecting characteristic in-cylinder flow features, Van Dam et al. [12] calculated the differences between the mean velocity magnitude and the standard deviations over the entire field of view to validate LES simulations of a GDI engine using experimental data. 
In order to quantify the flow variability of both experiments and LES simulations, Van Dam et al. [13] introduced the Circular Standard Deviation (CSD). This quantity is more appropriate for angular data than the regular standard deviation. In this way, variations in the direction of velocity components were separated from variations in the magnitude; a distinction that cannot be made by only comparing the RMS values of the velocity. This metric was also used to compare flow fields produced by sequential and parallel LES simulations [14].

A common metric for quantifying the differences in direction between two velocity vectors is the Relevance Index (RI), which was defined by Liu et al. [15]:

$$
R I=\frac{\left(\boldsymbol{q}_{\boldsymbol{A}}, \boldsymbol{q}_{\boldsymbol{B}}\right)}{\left|\boldsymbol{q}_{\boldsymbol{A}}\right|\left|\boldsymbol{q}_{\boldsymbol{B}}\right|}
$$

where $\left(\boldsymbol{q}_{\boldsymbol{A}}, \boldsymbol{q}_{\boldsymbol{B}}\right)$ denotes the inner product of vectors $\boldsymbol{q}_{\boldsymbol{A}}$ and $\boldsymbol{q}_{\boldsymbol{B}}$ and $\left|\boldsymbol{q}_{A}\right|$ denotes the magnitude of the vector $\boldsymbol{q}_{A}$. The RI returns the cosine of the angle between two vectors, and so produces values that range from -1 for completely mis-aligned vectors and 1 for perfectly aligned vectors. Therefore, the RI allows the difference in alignment between two vectors to be quantified by a single absolute value. This can be applied to a pair of velocity fields to produce a field of RI, which can be spatially averaged to obtain a single value that gives a measure of how closely two velocity fields are aligned. It has been used like this to directly compare simulated and measured flow fields [12][16].

An interesting use of the RI is to investigate the convergence of both simulated and experimental flow fields. Hu et al. [17] uses the RI (named the Structure Similarity Index) to find how many LES realisations are required to produce a representative mean for simulation of a turbulent, non-reacting jet. Similarly, Kuo et al. [18] used the RI to investigate the convergence of LES engine simulations by quantifying the variation of both the mean and RMS velocity magnitude fields for ensemble averages of varying numbers of cycles.

The RI has also been used to quantify the differences in modes produced by Proper Orthogonal Decomposition of flow fields in a GDI engine by Chen et al. [19][20] and has been applied to scalar fields such as the pixel intensity of spray images to investigate the variation in fuel spray characteristics [21][22].

An alternative metric, the Magnitude Similarity Index (MSI) was defined by $\mathrm{Hu}$ et al. [17]:

$$
M S I=1-\frac{\left|\boldsymbol{q}_{\mathrm{A}}-\boldsymbol{q}_{\mathrm{B}}\right|}{\left|\boldsymbol{q}_{\mathrm{A}}\right|+\left|\boldsymbol{q}_{\mathrm{B}}\right|}
$$

It produces a value of 1 if the vectors are identical and 0 if the vectors are exactly opposite. Unlike the Relevance Index, the MSI accounts for differences in both alignment and magnitude between two vector fields. This has been used by Van Dam et al. [14] and Ameen et al. [8] to directly quantify the differences between simulated and experimental flow fields. In a similar way to the RI, it has been used by Ameen et al. [23] to investigate the convergence of simulated scalar fields in a simulation of an n-dodecane fuel spray.

The metrics discussed above provide methods of quantifying the differences between flow fields, both simulated and experimental. However, typical flows setup during the induction stroke in a modern GDI engine are characterised by strong tumble motion [24-26]. Using the Relevance Index to compare flow fields with a vortex can lead to misleading results due to the regions of low velocities around the vortex centre. This work applies new metrics, the Weighted Relevance Index (WRI) and Weighted Magnitude Index (WMI), that robustly

Page 2 of 14 quantify the differences between velocity fields in terms of both magnitude and direction. These metrics are normalized and combined to produce a metric, the Combined Magnitude and Relevance Index (CMRI), that produces a single value which accounts for differences in both magnitude and direction. These metrics are used to assess the differences between velocity fields measured using high-speed PIV in an optical GDI engine and those simulated using RANS CFD for a range of test conditions.

\section{Experimental Setup}

\section{Optical Engine}

For this work, a single-cylinder GDI engine with optical access has been used. It has a pent-roof with 4 valves and a centrally mounted fuel injector. This study only considers motored flow; therefore, the spark plug has been replaced with a metal blank to reduce background scatter for the PIV measurements. Table 1 gives the engine specification.

Table 1. Engine Specification

\begin{tabular}{|l|l|}
\hline Bore $(\mathrm{mm})$ & 85 \\
\hline Stroke $(\mathrm{mm})$ & 90.3 \\
\hline Cylinder capacity $(\mathrm{cc})$ & 512 \\
\hline Compression ratio & 9.8 \\
\hline Valves per cylinder & 2 intake, 2 exhaust \\
\hline Injector & Centrally mounted \\
\hline Intake air temperature $(\mathrm{K})$ & 318 \\
\hline Coolant temperature $(\mathrm{K})$ & 295 \\
\hline
\end{tabular}

A LabView ${ }^{\mathrm{TM}}$ system has been used for data acquisition and synchronization of the timing of the PIV system and the engine measurements. Intake, exhaust and in-cylinder pressures were measured with Kistler pressure transducers and logged at a rate of 1 sample per degree crank angle. A programmable ECU was used to control the variable valve actuation system and the intake throttle to ensure repeatable boundary conditions.
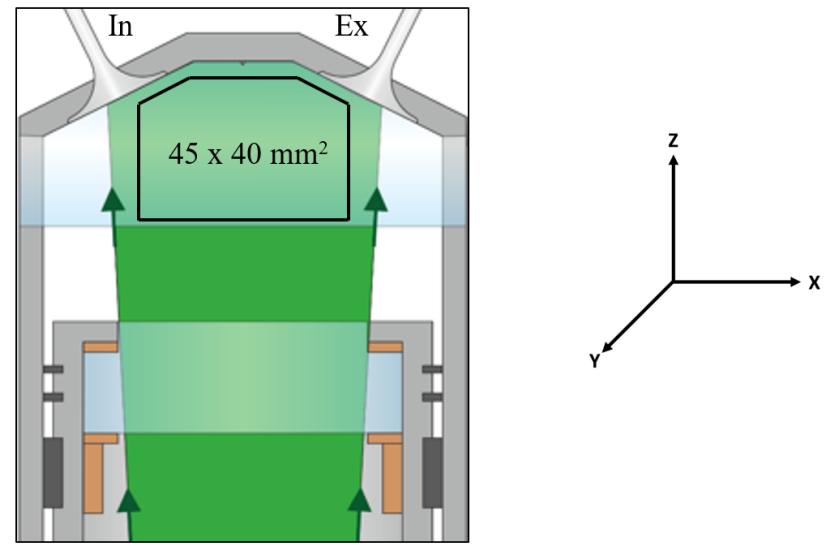

Figure 1. An image showing the location of the laser sheet in the optical engine, PIV field of view and coordinate system.

A transparent acrylic annulus with a curved top surface was used to provide an uninterrupted view of the combustion chamber. An 
extended Bowditch piston arrangement with a quartz piston insert and a $45^{\circ}$ mirror provided access for the illuminating laser sheet. The piston crown had shaped features around the circumference that limited the width of the piston window to $45 \mathrm{~mm}$ and blocked the view of the combustion chamber at TDC.

\section{PIV Setup}

PIV measurements were made every $5^{\circ} \mathrm{ca}$ from $330^{\circ} \mathrm{ca} \mathrm{bTDC}$ (relative to combustion TDC) to $30^{\circ} \mathrm{ca}$ bTDC to capture both the induction and compression strokes. Measurements near TDC were unavailable due to the piston crown features blocking the scattered light from the oil droplets. The measurement plane was located $1 \mathrm{~mm}$ towards the front of the engine from the central tumble plane $(y=-1 \mathrm{~mm}$ using the coordinate system in Figure 1). This was to reduce the large amount of scattered laser light from the tip of the fuel injector affecting the measurement region.

The seeding system consisted of a LaVision aerosol generator that produced micron-sized droplets of vegetable oil, which entered the airpath via a large plenum upstream of the inlet valves. The seeding density was controlled by a mass flow controller that measured the mass flow of air entering the atomizer. For each test condition, the optimal seeding density was obtained using an iterative process.

A diode-pumped, double cavity Nd:YLF laser (Photonics Industries DM20-527DH) was used to provide illumination for the oil droplets. Dependent on the engine speed, the laser was operated at a range of frequencies from $1.8 \mathrm{kHz}(1500 \mathrm{rpm})$ to $2.1 \mathrm{kHz}(1750 \mathrm{rpm})$. The beam was formed into a sheet of approximately $1 \mathrm{~mm}$ thickness in the measurement region using an arrangement of spherical lenses and a cylindrical lens with focal length of $-20 \mathrm{~mm}$.

A high-speed, 12-bit digital CMOS camera (Vision Research VEO $710 \mathrm{~L}$ ) was used to image the scattered light from the oil droplets. A Nikon Nikkor $50 \mathrm{~mm} \mathrm{f/1.4} \mathrm{lens} \mathrm{was} \mathrm{used} \mathrm{with} \mathrm{an} \mathrm{aperture} \mathrm{setting} \mathrm{of}$ $\mathrm{f} / 4$ to balance the brightness of the oil particle images, scatter from engine surfaces and the depth of field. The sensor resolution of $1020 \times 800$ pixels resulted in an approximate pixel resolution of $55 \mu \mathrm{m} /$ pixel.

A LaVision Programmable Timing Unit (PTU v10) was used to synchronise the timings of the laser and camera triggers. It also has the ability to vary the delay between the two laser pulses, $\mathrm{d} t$, within a cycle. There is a large variation in flow velocities from the intake process to the compression stroke, which results in variation of the optimal $\mathrm{d} t$ [27]. Therefore, the optimal $\mathrm{d} t$ 's were obtained iteratively for each test condition by ensuring that the particle displacement does not exceed a quarter of the length of the final interrogation window size ( 8 pixels for a window of 32 pixels). For a typical test condition, the $\mathrm{d} t$ can range from $3 \mu$ s to $50 \mu$ s throughout the cycle.

LaVision DaVis ${ }^{\mathrm{TM}}$ 8.0 PIV software was used to operate the PIV equipment and process the raw images. Any image distortion caused by the curved surface of the transparent annulus was corrected by using a calibration image with a grid of uniformly spaced dots. This also provided the mapping of the image to a real-world coordinate system. The intensity of the raw particle image pairs was first normalized using a min-max filter, before a static digital mask removed regions of the image that were not used in the velocity calculation (e.g. the cylinder head and regions outside the laser sheet). A multi-pass crosscorrelation algorithm with adaptive window deformation and shift was used for the velocity calculation. The first pass used an interrogation window of $128 \times 128$ pixels, and this decreased each pass resulting in a final window size of $32 \times 32$ pixels, for which there were three passes. The final window size corresponded to a spatial resolution of $1.92 \mathrm{~mm}$, and with $50 \%$ overlap, a vector spacing of $0.96 \mathrm{~mm}$.

A validation routine was used to remove any spurious vectors from the final vector field used in the analysis. Any vector with a correlation peak ratio $Q$ less than 2 was deleted, where $Q$ is the ratio of the highest correlation peak to the second highest peak in the interrogation window. Only $1^{\text {st }}$ choice vectors, which accounted for approximately $99 \%$ of all vectors, were used for the analysis. The universal outlier detection method as outlined by Westerweel et al. [28] was used for further detection of spurious vectors.

MATLAB ${ }^{\mathrm{TM}}$ was used for any further processing, such as adding additional masking to remove features that obscure the field-of-view during the cycle, such as the intake valve and the piston.

\section{Numerical Setup}

A commercially available CFD package, StarCD, was used for the RANS simulations of the GDI engine. The computational domain for the simulations is shown in Figure 2.

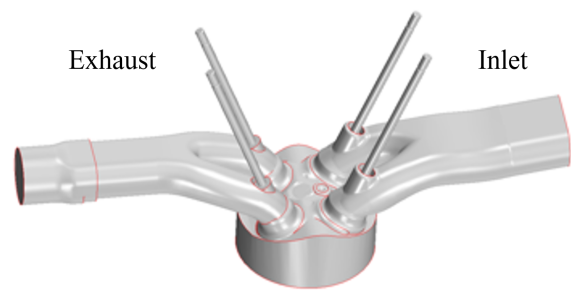

Figure 2. Image of the computational domain used for the RANS simulations.

Experimental data from the optical engine were used as boundary conditions for the simulations; at the intake and exhaust ports temperature and dynamic pressure measurements were used as inputs. The mass flow of air entering the engine and the valve lift profiles were also matched to experimental conditions.

Dynamic meshing was used, and the mesh had a minimum cell size of $0.7 \mathrm{~mm}$ but the general mesh size for the valve curtain area was $0.3 \mathrm{~mm}$; each simulation contained approximately 3.25 million cells. The timestep for the simulation was $0.1^{\circ} \mathrm{ca}$. Turbulence was modelled using the standard RNG k- $\varepsilon$ model and a modified Angelberger model was used to model heat transfer.

For these simulations, to match the in-cylinder pressure to experiments, the crevice length (defined as the distance between the top land and the top piston ring) was varied for each condition. There was no further tuning of the physical models. The measured and simulated pressure traces are shown in Figure 3. As the minimum cell size was $0.7 \mathrm{~mm}$, the CFD package used linear interpolation to output the values of the vectors at the locations of the PIV measurements.

\section{Description of Quantitative Metrics for Comparison of Vector Fields}

This section describes three related metrics that have been developed for application to flow fields typical of in-cylinder charge motion in a modern GDI engine. These metrics have been developed to produce robust, quantitative comparisons between flow fields in terms of alignment and magnitude.

Page 3 of 14 


\section{Weighted Relevance Index (WRI)}

The relevance index, defined in Equation (1), has no dependence on the magnitudes of the vectors being compared. As a result, the alignment of low velocity vectors carries the same weight as that of high velocity vectors, which can lead to potentially misleading spatially averaged RI values for flow fields containing vortex structures. The combination of small velocities near the vortex centre and the reversal of the flow direction across the centre of the vortex causes even very small differences in the vortex centre location to result in small regions of very misaligned vectors with very high RI values. Although these vectors are indeed misaligned, their contribution to the spatially averaged RI can be disproportionate to the overall alignment of the two flows as the absolute velocity difference near the vortex centre is small. Therefore, a small offset in the location of the vortex centres can dominate the evaluation of spatially averaged RI fields, potentially masking other differences between the flow fields.

The Weighted Relevance Index (WRI) introduced in this work provides an alternative metric for quantifying vector alignment which weights the contribution of each vector pair to an 'alignment penalty' by the ratio of the local velocities to the medians of the velocity magnitudes present within each vector field. The WRI is defined as:

$$
\begin{aligned}
& \boldsymbol{W} \boldsymbol{R} \boldsymbol{I}\left(x_{i}, z_{j}\right)=\ldots \\
& \qquad\left(\frac{1-\boldsymbol{R I}\left(x_{i}, z_{j}\right)}{2}\right)\left(\frac{\boldsymbol{Q}_{A}\left(x_{i}, z_{j}\right)}{\operatorname{median}\left(\boldsymbol{Q}_{A}(x, z)\right)}\right)\left(\frac{\boldsymbol{Q}_{B}\left(x_{i}, z_{j}\right)}{\operatorname{median}\left(\boldsymbol{Q}_{B}(x, z)\right)}\right)
\end{aligned}
$$

where $\boldsymbol{Q}_{A}(x, z)$ is the magnitude field of vector field $\boldsymbol{q}_{\boldsymbol{A}}(x, z)$ such that $\boldsymbol{Q}_{A}\left(x_{i}, z_{j}\right)=\left|\boldsymbol{q}_{A}\left(x_{i}, z_{j}\right)\right|$ with spatial coordinates $x_{i}$ and $z_{i} . \boldsymbol{R I}\left(x_{i}, z_{j}\right)$ is the Relevance Index as defined in Equation (1).

The velocity weighting for each field suppresses the alignment penalty for individual vectors whose velocities are small compared to their own field. In this way, the sensitivity to the misalignment of the small velocity vectors near the centre of a vortex is avoided, while global changes to the velocity magnitudes across either field do not affect the WRI values. For perfectly aligned vectors, the misalignment penalty of Equation (3) is zero, giving a WRI value of zero for any velocity magnitude. For a given magnitude, increasing misalignment results in increasing values of the WRI. The largest values of the WRI are the result of poorly aligned, high velocity vectors.

\section{Weighted Magnitude Index (WMI)}

In a similar approach to the WRI, a weighting factor is included in the definition of the WMI (Equation (4)) to scale the contributions of the differences in local velocity magnitudes by the average speed of the flow field. The WMI is defined as:

$$
\boldsymbol{W M I}\left(x_{i}, z_{i}\right)=\frac{\left|\boldsymbol{Q}_{A}\left(x_{i}, z_{j}\right)-\boldsymbol{Q}_{B}\left(x_{i}, z_{j}\right)\right|}{\operatorname{median}\left(\boldsymbol{Q}_{A}(x, z), \boldsymbol{Q}_{B}(x, z)\right)}
$$

where the weighting factor in the denominator is the median velocity magnitude for all velocity magnitudes in vector fields $\boldsymbol{A}$ and $\boldsymbol{B}$.

Similarly to the WRI, the WMI returns zero for vectors with identical magnitudes and high values for large vectors with large velocity differences. The median velocity magnitude is used in the definitions of the both the WRI and WMI as it is less affected by extreme values than the mean, which results in a more robust weighting.

\section{Combined Magnitude and Relevance Index (CMRI)}

Differences in alignment between two flow fields are not necessarily correlated with differences in magnitude. In order to quantitatively determine the similarity of two vector fields it is useful to define a single metric that accounts for the differences in both alignment and magnitude. For the flow fields investigated in this work, the WRI and WMI typically have very different scales, with the distribution of the WRI values more tightly grouped around zero than those of the WMI due to the cosine in the definition of the Relevance Index. Simple combinations of the two metrics often result in the WRI having a negligible contribution to the combined metric.

To achieve approximately equal weighting between the WRI and WMI contributions, the WRI and WMI values are each linearly scaled such that $96 \%$ of the values for each metric lie between 0 and 1 to give normalised metrics, the $\mathrm{WRI}_{\text {norm }}$ and $\mathrm{WMI}_{\text {norm }}$. The value of $96 \%$ was chosen as this corresponds to just over two standard deviations away from the mean in a normal distribution. For a typical pair of vector fields in this work, $96 \%$ of WMI values lie between 0 and 0.7 , compared to the WRI where $96 \%$ of the values lie between 0 and 0.1 . The normalised versions of each metric redistribute these values over the range 0 to 1 . The Combined Magnitude and Relevance Index (CMRI) is defined as the mean of the normalised WRI and WMI metrics:

$$
\operatorname{CMRI}\left(x_{i}, z_{i}\right)=\frac{\left(\boldsymbol{W R I _ { \text { norm } }}\left(x_{i}, z_{i}\right)+\boldsymbol{W M I}_{\text {norm }}\left(x_{i}, z_{i}\right)\right)}{2}
$$

The CMRI provides a complimentary alternative to the more specific WRI and WMI. It provides a method of identifying general differences between flow fields, however, for further analysis of the cause of these differences, the WRI and WMI must also be used. The CMRI, WRI and WMI therefore provide a set of metrics, suited to in-cylinder flows, which should be applied in combination for quantitative comparison of vector fields.

\section{Testing}

Table 2 summarises the three test conditions that are investigated in this study.

Table 2. Summary of test conditions

\begin{tabular}{|l|l|l|l|l|l|}
\hline $\begin{array}{l}\text { Test } \\
\text { condition }\end{array}$ & $\begin{array}{l}\text { Engine } \\
\text { speed } \\
(\mathrm{rpm})\end{array}$ & $\begin{array}{l}\text { Intake } \\
\text { pressure } \\
(\mathrm{kPa})\end{array}$ & $\begin{array}{l}\dot{V}_{a} \\
(\mathrm{~L} / \mathrm{s})\end{array}$ & $\begin{array}{l}\text { IVO/IVC } \\
\left({ }^{\circ} \mathrm{ca} \text { aTDC }\right)\end{array}$ & $\begin{array}{l}\text { EVO/EVC } \\
\left({ }^{\circ} \mathrm{ca} \text { aTDC) }\right.\end{array}$ \\
\hline $\mathrm{T} 1$ & 1500 & 80 & 1.57 & $327 /-259$ & $143 /-309$ \\
\hline $\mathrm{T} 2$ & 1500 & 95 & 3.65 & $330 /-243$ & $117 /-335$ \\
\hline $\mathrm{T} 8$ & 1750 & 96 & 3.4 & $337 /-248$ & $141 /-311$ \\
\hline
\end{tabular}

Figure 3 shows plots of both the experimental and simulated cylinder pressure traces for each test condition, as well as the inlet valve lift profiles. 

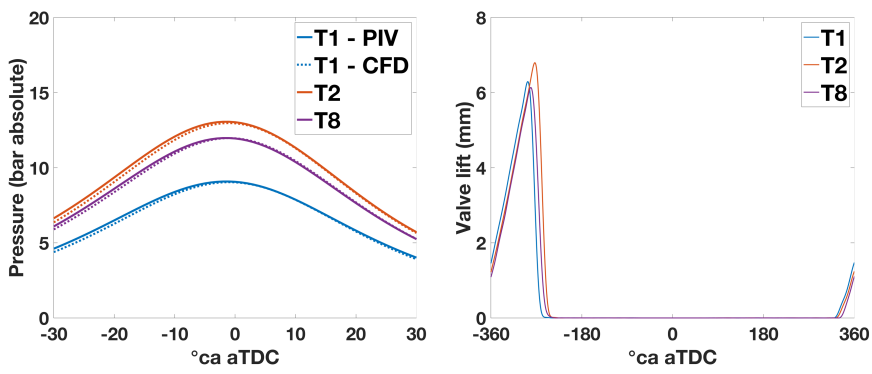

Figure 3. Simulated and experimental cylinder pressure traces (left) and intake valve lifts (right).

The engine is operated at $1500 \mathrm{rpm}$ for $\mathrm{T} 1$ and $\mathrm{T} 2$, and at $1750 \mathrm{rpm}$ for T8. T2 and T8 are both operated close to wide open throttle, whereas the MAP was reduced to $80 \mathrm{kPa}$ for $\mathrm{T} 1$, as reflected in the pressure traces.

CFD simulations provide a three-dimensional, three-component velocity field, which must be reduced to a planar field in order to directly compare with PIV results. Firstly, it was ensured that the geometric origin of the simulations and the PIV measurements were identical. Two-component CFD vectors from the plane of interest $(y=-1 \mathrm{~mm})$ were then extracted onto a grid that had twice the spatial resolution as the PIV measurement grid. The resulting CFD vector field was interpolated before it was then mapped directly onto the PIV measurement grid. As result of this process, both simulated and measured flow fields were located on identical spatial grids. An example of a CFD flow field mapped onto a PIV measurement grid is shown in Figure 4.
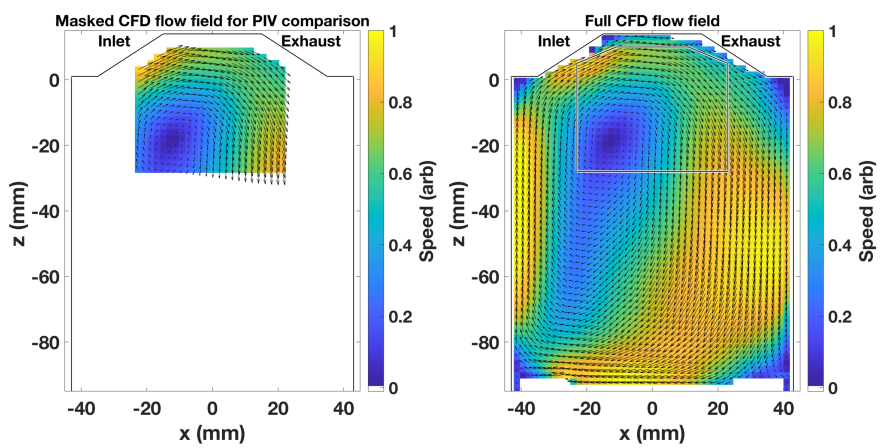

Figure 4. Example of a CFD flow field (right) mapped onto the PIV measurement grid (left). The box on the right-hand figure shows the field of view of the PIV measurement.

The PIV flow fields used for the validation of the RANS simulations are averages of 300 cycles recorded in three sets of 100 cycles. In a previous study, this number of cycles was shown to be sufficient for a representative mean cycle [29].

It should be noted that both PIV and CFD vector fields were processed with an identical digital mask to remove vectors from the inlet valve and piston. Due to the different valve profiles, the most conservative mask (for T2) was used for all conditions for a fair comparison. All flow fields plotted in the Results section show on every second vector for clarity and arbitrary units are adopted.

\section{Results}

This section demonstrates how the quantitative metrics described previously can be used to identify differences between the CFD and Page 5 of 14
PIV flow fields. A number of differences identified by the metrics are then explored in more detail using conventional methods of comparison to evaluate the performance of the metrics.

\section{Initial Investigation Using the CMRI, WRI and WMI}

Figure 5 shows plots of the spatially averaged CMRI throughout the cycle for all three test conditions, which provide initial comparisons between simulated and measured flow fields.

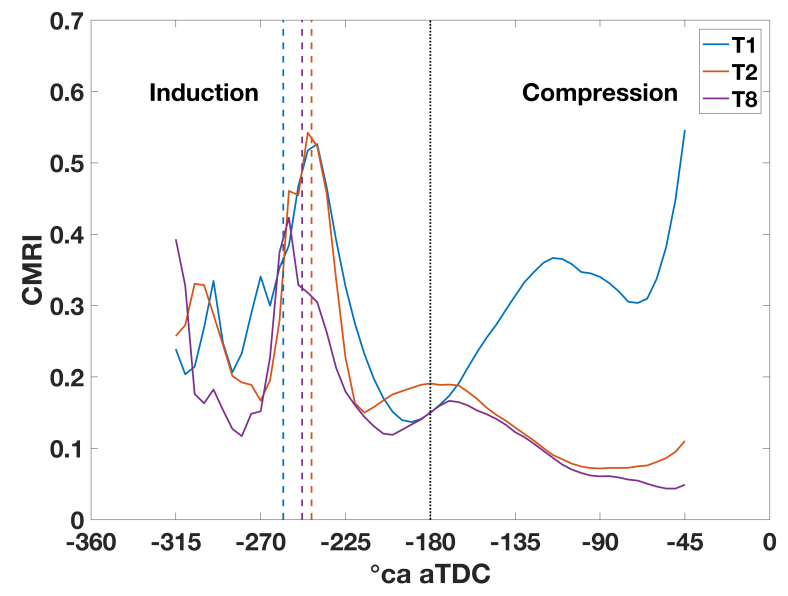

Figure 5. Plot of the spatially-averaged CMRI showing the differences between simulated and measured flow fields in terms of alignment and magnitude. The dashed lines show the timing of IVC for each condition. Low values denote a better match between flow fields.

There are a number of features that can be highlighted from this plot; firstly, there are large peaks in the CMRI that occur at approximately $240^{\circ} \mathrm{ca}$ bTDC for T1 and T2. There is also a peak present for T8, but it is less well defined and smaller in magnitude than T1 and T2. Shortly after BDC, the CMRI for T2 and T8 decreases to a minimum during the compression stroke. In contrast, the CMRI for T1 increases significantly until approximately $110^{\circ} \mathrm{ca}$ bTDC. After a local minimum at $70^{\circ} \mathrm{ca} \mathrm{bTDC}$, the match between CFD and PIV flow fields for $\mathrm{T} 1$ deteriorates rapidly, whereas there are only small changes in the quality of the match between CFD and PIV flow fields for T2 and T8.

The plot of the CMRI allows regions of interest to be easily identified in the comparison between two flow fields. As it accounts for differences in both alignment and magnitude of the velocity fields, further analysis of the WRI and WMI metrics is required to decouple their contributions to the CMRI. Figure 6 shows plots of the WRI and WMI for all conditions. For the remainder of this paper, the normalised WRI and WMI are presented.

Inspecting the WRI and WMI separately provides more information about nature of the features identified by the CMRI (Figure 5). For example, by examining the plot of the WRI, it is clear that the peaks in the plots of the CMRI at $240^{\circ} \mathrm{ca}$ bTDC for all conditions is due to a misalignment of the flow fields, rather than a difference in magnitude. Furthermore, the plot of the WMI reveals a number of peaks that correspond to the inlet valve closing period for each condition. These peaks are also visible in the plot of the CMRI, however it is less clear due to the contribution of the WRI at around $240^{\circ} \mathrm{ca}$ bTDC. The increase in the CMRI for T1 during the compression stroke from BDC to $110^{\circ} \mathrm{ca}$ bTDC appears to be a combination of both a misalignment and difference in magnitude between simulated and measured vector fields. 

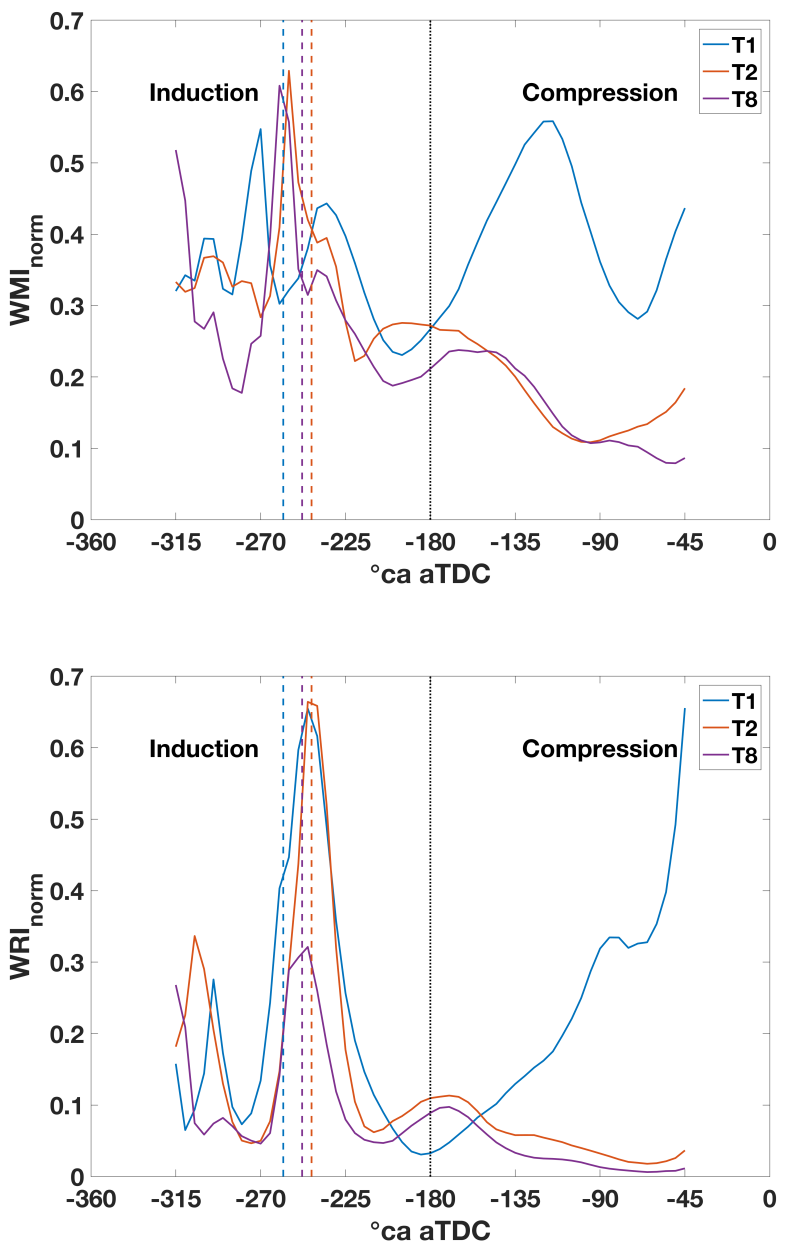

Figure 6. Plot of the spatially averaged WMI (top) and WRI (bottom) showing the differences between simulated and measured flow fields in terms of magnitude and alignment separately. The coloured dashed lines show the timing of IVC for each condition.

Three features identified by the metrics, which will be discussed in more detail are listed below:

- $\quad$ Peaks in the WMI during inlet valve closing

- Peaks in the WRI for all conditions at $240^{\circ} \mathrm{ca}$ bTDC

- Increase in all metrics during the compression stroke for T1

\section{Peaks in the WMI During Inlet Valve Closing}

Figure 6 shows distinct peaks of the WMI at timings that correspond to the period of inlet valve closing for each condition. To investigate the origin of these peaks, fields of the WMI for each condition at the respective timings of the peaks of the WMI are displayed in Figure 7. The peaks occur at $270^{\circ} \mathrm{ca} \mathrm{bTDC}, 255^{\circ} \mathrm{ca} \mathrm{bTDC}$ and $260^{\circ} \mathrm{ca} \mathrm{bTDC}$ for $\mathrm{T} 1, \mathrm{~T} 2$ and $\mathrm{T} 8$ respectively. The simulated and experimental velocity vectors are overlaid on the values of the WMI to show both flow fields.
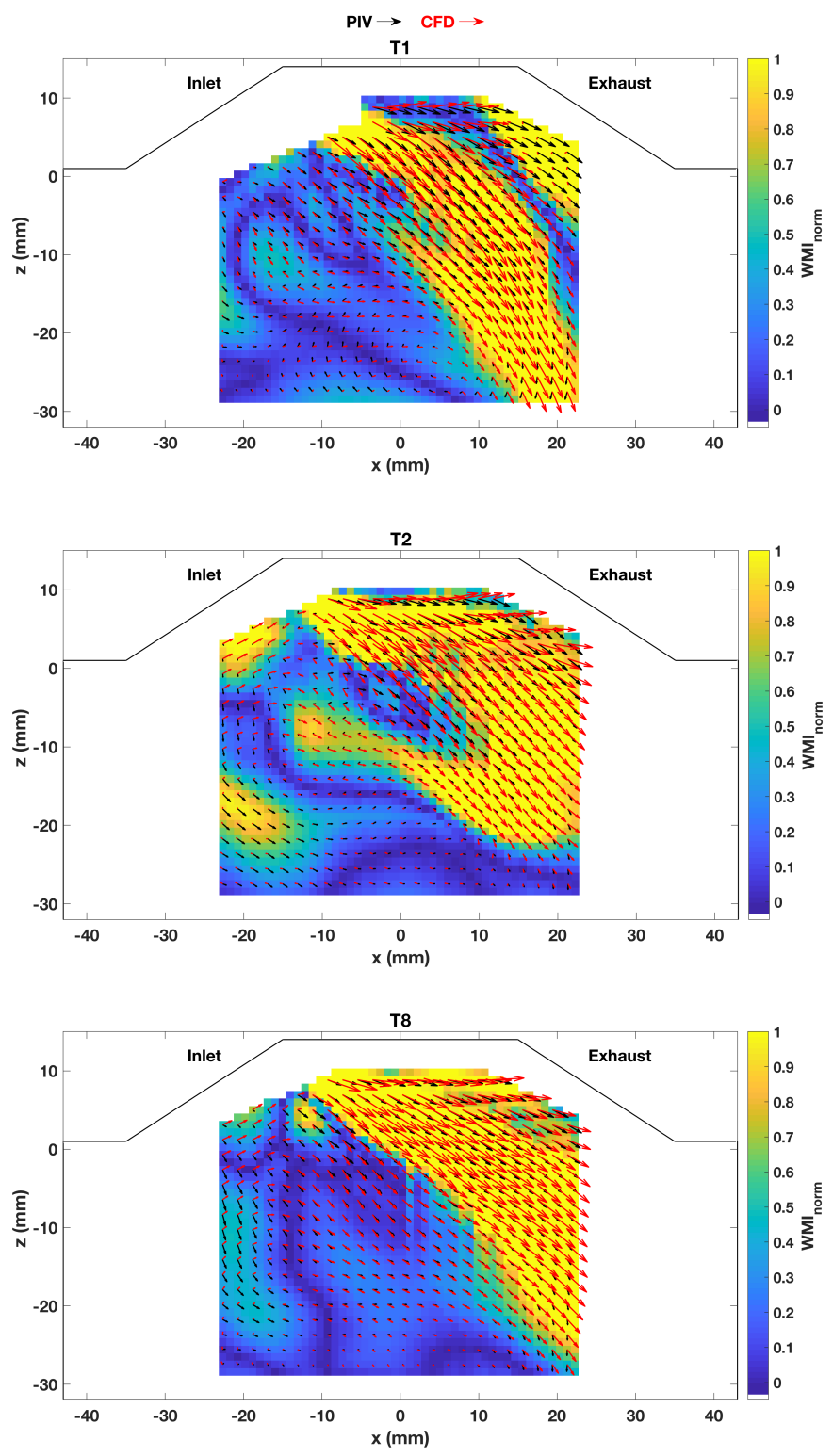

Figure 7. Fields of the WMI at the locations of the peaks shown in Figure 6. $\mathrm{T} 1-270^{\circ} \mathrm{ca} \mathrm{bTDC}, \mathrm{T} 2-255^{\circ} \mathrm{ca} \mathrm{bTDC}$ and T $-260^{\circ} \mathrm{ca} \mathrm{bTDC}$. The overlaid black and red arrows show the PIV and CFD vector fields respectively.

In this period, as the inlet valve is closing, there is a high-speed jet from the inlet valve entering the combustion chamber for all conditions. In this region, there are relatively high values of the WMI between the two flow fields. On inspection of the velocity vectors, it is clear that the simulations predict higher velocities in the jet than the PIV results for all conditions, which leads to high values of the WMI. Although there are differences in the velocities on the inlet side of the field of view, they are relatively small compared to those in the jet and so result in small values of the WMI.

The higher momentum of the simulated intake flow results in the intake jet penetrating further into the cylinder than the experimental results. This can be seen in the lower, right-hand side of the field of view, where the simulated flow field persists in the direction of the inlet jet. In contrast, the PIV flow fields begin to turn towards the centre of the cylinder due to a loss of momentum. It is worth noting that the PIV flow fields are averages of 300 individual cycles, therefore any 
variations in the intake flow will smoothed out. There are indeed single PIV cycles for which the magnitude of the velocity in the intake jet is more similar to that simulated by CFD

One metric that is commonly used to assess the performance of modern GDI engines is the tumble ratio (TR). This can be defined in a number of ways, but for this study, the method defined by StarCD [29] has been adopted. This is given by:

$$
T R=\frac{\sum_{N}\left[\left(Z_{i}-Z_{m}\right) u_{i}-\left(X_{i}-X_{m}\right) w_{i}\right]}{\sum_{N} \sqrt{\left(Z_{i}-Z_{m}\right)^{2}+\left(X_{i}-X_{m}\right)^{2}}}
$$

where $N$ is the number of vectors in the velocity field, $X_{i}$ and $Z_{i}$ define the spatial location of the velocity vector of two components, $u_{i}$ and $w_{i}$, and $X_{m}$ and $Z_{m}$ define the spatial location of the centre of mass (which is equivalent to the centre of volume as density is assumed to be constant throughout the cylinder). This definition produces a single value that is positive for a velocity field that has a clockwise rotational direction for the coordinate system used in this study. Tumble ratio is dependent on the magnitude of the velocity vectors, their direction and their location in relation to the centre of mass. Figure 8 shows plots of TR for all three conditions, with dotted lines marking the timings of peak valve lift and valve closing.

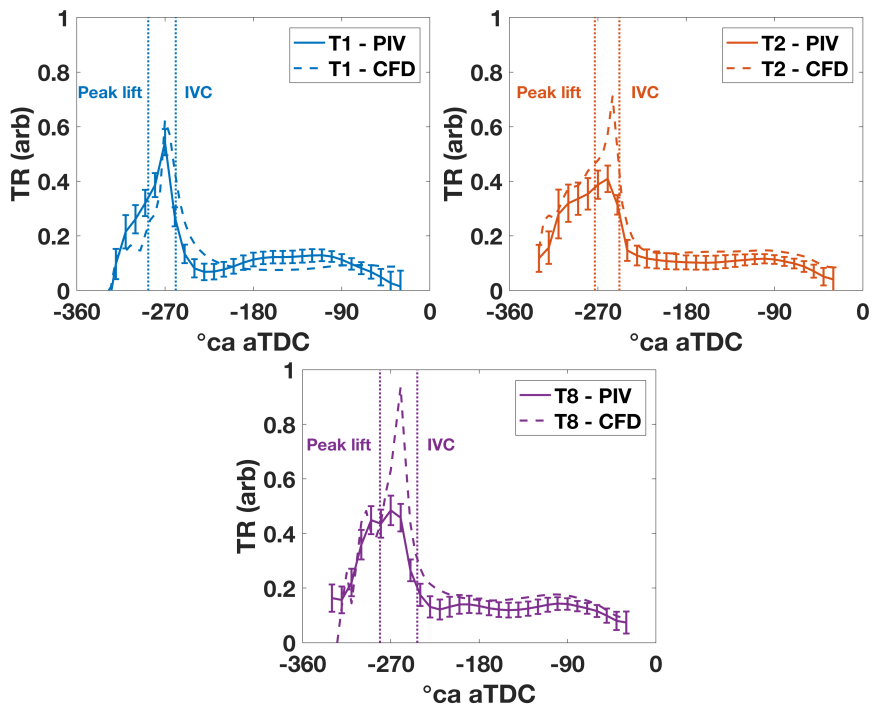

Figure 8. Plots of tumble ratio (TR) for T1, T2 and T8. The dotted lines mark the timings of peak valve lift and valve closing. The error bars show $+/-1$ standard deviation for the TR for every $10^{\text {th }}$ crank angle of the PIV flow fields.

The main difference between the CFD and PIV results in Figure 8 is the large peak in the simulated TR values that occurs during the valve closing period for all conditions. These peaks correspond directly to the peaks of the spatially averaged WMI identified in Figure 6, which leads to the conclusion that the difference in TR between PIV and CFD is due to differences in the speed of the intake jet, as discussed previously.

This definition of tumble ratio is dependent on the direction of the velocity vectors, in addition to their location and magnitude. Therefore, the differences between CFD and PIV could also be due to a mis-alignment of the intake jet. However, the overlaid flow fields in Figure 7 are qualitatively well-aligned in the region of the inlet jet and fields of the WRI (not shown here) quantitatively agree.
It is evident that the difference in TR between CFD and PIV is significantly higher for $\mathrm{T} 8$ and $\mathrm{T} 2$ than for $\mathrm{T} 1$. On inspection of the CFD flow fields in Figure 7 (red arrows), the high-speed flow of the intake jet for T2 and T8 fills the entire upper, right-hand side of the field of view. In contrast, the CFD flow field for T1 has a stagnant region near the exhaust side of the cylinder head. This appears as a high value of the WMI because there are large differences between PIV and CFD velocity magnitudes in this region, as the PIV results do not have this region of slow flow. However, the stagnant region in the simulation results in a reduced value of TR, which agrees more closely with experiment, even though there is a difference in the structure of flow fields.

\section{Peaks in the WRI at $240^{\circ}$ ca bTDC}

Dominant peaks at $240^{\circ} \mathrm{ca}$ bTDC in the plots of the CMRI (Figure 5) also feature in the plots of the WRI shown in Figure 6 (bottom). These peaks occur for all conditions; however, they are noticeably larger for $\mathrm{T} 1$ and T2 in comparison to T8. It is worth noting that at this timing, the inlet valve has closed for all conditions. To investigate the cause of such differences in the flow fields between CFD and PIV, Figure 9 presents fields of the WRI for each condition at $240^{\circ} \mathrm{ca}$ bTDC.

As expected from the spatially averaged results, the WRI fields demonstrate that there is a significantly greater misalignment between the simulated and experimental flow fields for T1 and T2, than for T8. It is interesting to note that, for all conditions, the simulations predict a very similar velocity field after IVC. Whereas, in contrast, the mean PIV flow fields for each condition display distinct differences. The simulated results show that after IVC, a strong tumble flow is setup for all conditions with some variation in the location of the main tumble vortex. Similarly, the experimental flow fields for T1 and T8 reveal a strong clockwise tumble motion. Although the main PIV tumble vortex is not visible in the experimental field of view at this time, by inspecting the direction of the vectors, it can be inferred that the tumble vortex for these points is located beyond the lower left-hand corner of the field of view. Relative to its corresponding simulation, there appears to be a greater offset between the location of the vortex centre for $\mathrm{T} 1$ than that of $\mathrm{T} 8$, which results in a greater misalignment for velocity vectors of greater magnitude. As discussed previously, the definition of the WRI (Equation (3)) penalises misaligned, high-speed vectors more than low-speed vectors. Around the centre of the CFD tumble vortex for both $\mathrm{T} 1$ and $\mathrm{T} 8$, the values of the WRI are relatively low, but as the distance from the vortex centre increases there is an increase in the weighting of the misalignment in the calculation of the WRI. The predicted and measured tumble vortices are closer for T8 than for T1; therefore, far away from the vortex centre, the high-speed vectors are more closely aligned, resulting in a lower WRI. In contrast, for $\mathrm{T} 1$, the offset between the tumble vortices is relatively large, which results in some high-speed velocity vectors 180 degrees misaligned and high values of the WRI. 

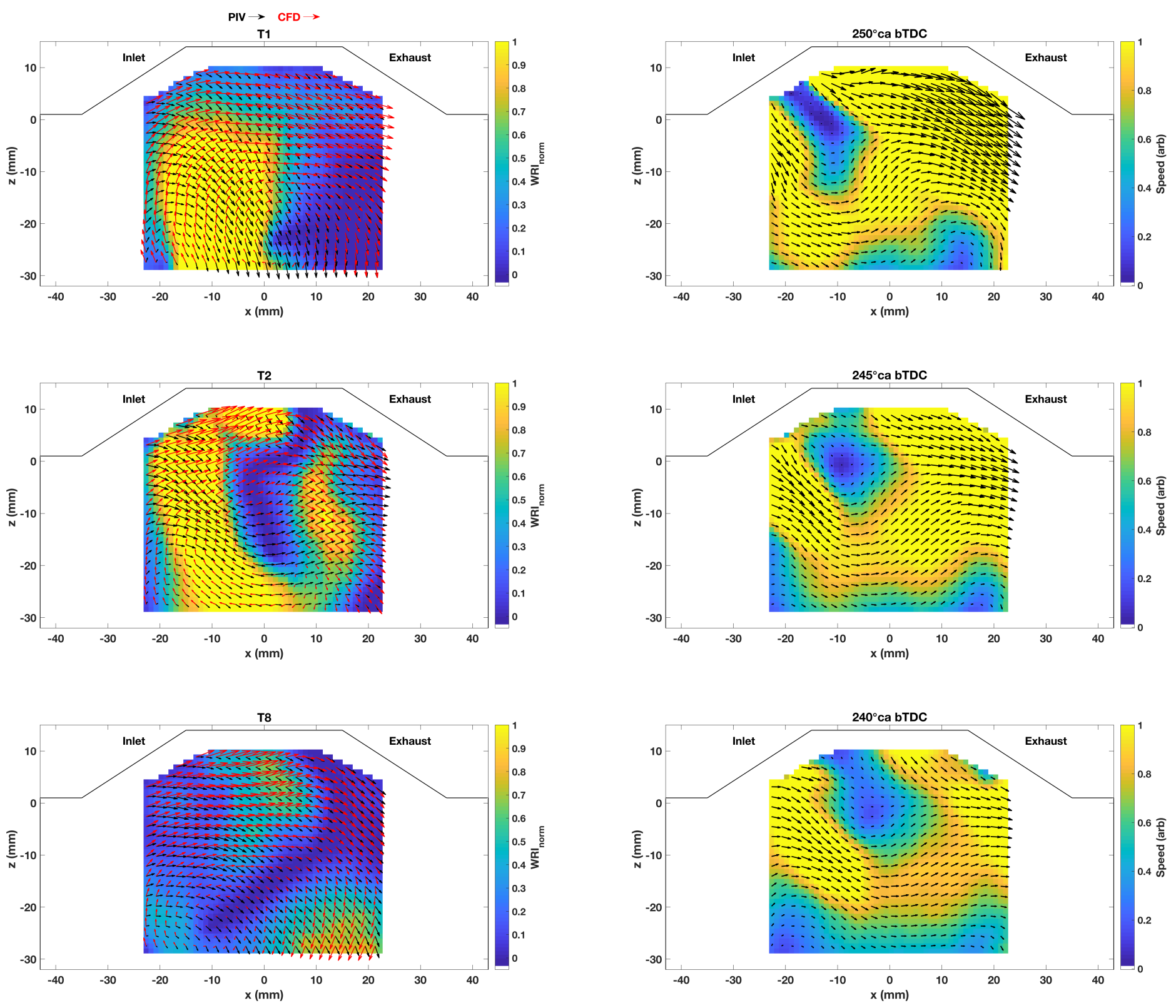

Figure 9. Fields of the WRI at $240^{\circ} \mathrm{ca}$ bTDC for T1, T2 and T8. The overlaid black and red arrows show the PIV and CFD vector fields respectively.

The measured flow field for $\mathrm{T} 2$ has a distinctly different structure to those of T1 and T8. Instead of a large-scale tumble motion, there is a region of low velocity flow in the centre of the combustion chamber that interrupts the tumbling motion and results in large differences in alignment between simulated and measured flow fields. On further analysis of the PIV flow fields, this feature appears for all conditions, but is dependent on the timing of IVC. For example, IVC for T1 occurs earlier than T2 and T8 (see Table 2 for details), therefore this feature appears earlier in the flow fields for T1. Figure 10 shows the evolution of the measured in-cylinder flow for $\mathrm{T} 2$ for a number of measurements after IVC.

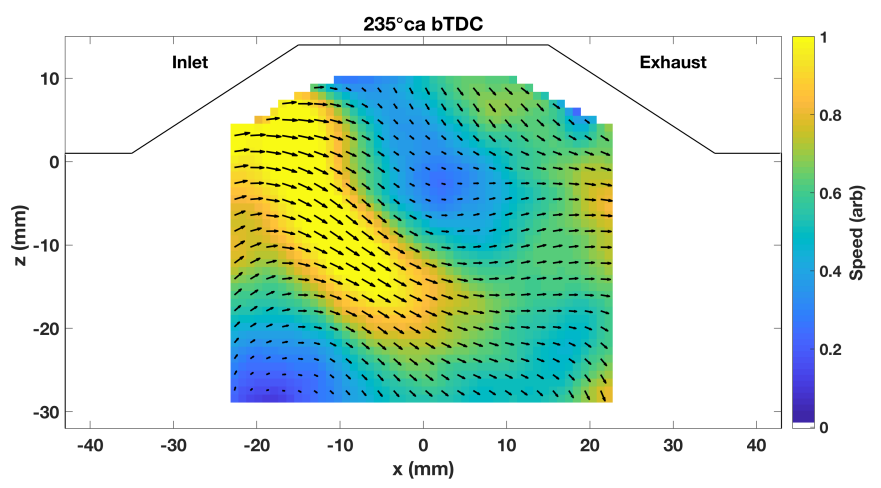

Figure 10. Mean PIV velocity fields showing the evolution of the flow field for T2 from $250^{\circ} \mathrm{ca} \mathrm{bTDC}$ to $235^{\circ} \mathrm{ca} \mathrm{bTDC} \mathrm{(IVC} \mathrm{occurs} \mathrm{at} 243^{\circ} \mathrm{ca} \mathrm{bTDC}$ ). 
IVC for T2 occurs at $243^{\circ} \mathrm{ca}$ bTDC, hence the flow field at $250^{\circ} \mathrm{ca}$ bTDC (Figure 10, top) shows the flow field as the inlet valve is closing. The region of slow flow is initially located near the inlet valve. The flow fields at $245^{\circ} \mathrm{ca} \mathrm{bTDC}, 240^{\circ} \mathrm{ca} \mathrm{bTDC}$ and $235^{\circ} \mathrm{ca}$ bTDC (Figure 10, from top to bottom) show how the region of slow flow moves towards the exhaust and grows, directing the main tumble motion towards the centre of the cylinder. This is in contrast to the simulations, which predict that the tumbling flow follows the boundary of the cylinder head directly after IVC.

Again, it must be noted that comparisons between mean PIV flow fields and CFD simulations hide the variation apparent in the PIV measurements of individual cycles. Therefore, small differences in the location of vortex centres between simulated and mean PIV flows does not necessarily suggest that the CFD is incorrect. There may be a single PIV cycle that better matches the simulation than the mean PIV flow field.

\section{Increase in All Metrics for T1 During the Compression Stroke}

The plot of the spatially averaged CMRI (Figure 5) shows that the difference between simulated and measured flow fields for $\mathrm{T} 1$ increases during the compression stroke until approximately $110^{\circ} \mathrm{ca}$ bTDC. The opposite is true for T2 and T8, for which the match improves during the same period. The plots of the WRI and WMI (Figure 6) confirm that over this period, the differences in both alignment and magnitude contribute to the increase in the CMRI.

Figure 11 shows plots of the WRI and WMI for T1 at $110^{\circ} \mathrm{ca}$ bTDC, as well as the corresponding CFD and PIV velocity fields. The PIV and CFD velocity fields shown in Figure 11 (c) and (d) show that the simulation underestimates the speed of the measured flow. The main tumble vortex for the PIV flow field is located near the bottom right-hand corner of the field of view, whereas the vortex centre for the simulated flow field is below the bottom edge of the field of view. These differences in the flow fields give rise to the increases in the WRI and WMI, and subsequently, the CMRI. The plot of the WRI shown in Figure 11 (a) shows a region of higher values slightly to the left of the PIV tumble vortex. This is due to misaligned vectors with relatively high velocities. At the centre of the PIV tumble vortex, the WRI values are low.

The plot of the WMI in Figure 11 (b) shows a region of high values on the inlet side of the measurement region. This corresponds to the region of biggest difference between flow speeds shown in the Figure 11 (c) and (d). The value of the WMI reduces towards the righthand side of the field of view as the absolute differences in magnitude are small compared to the overall flow speeds in the measurement region.
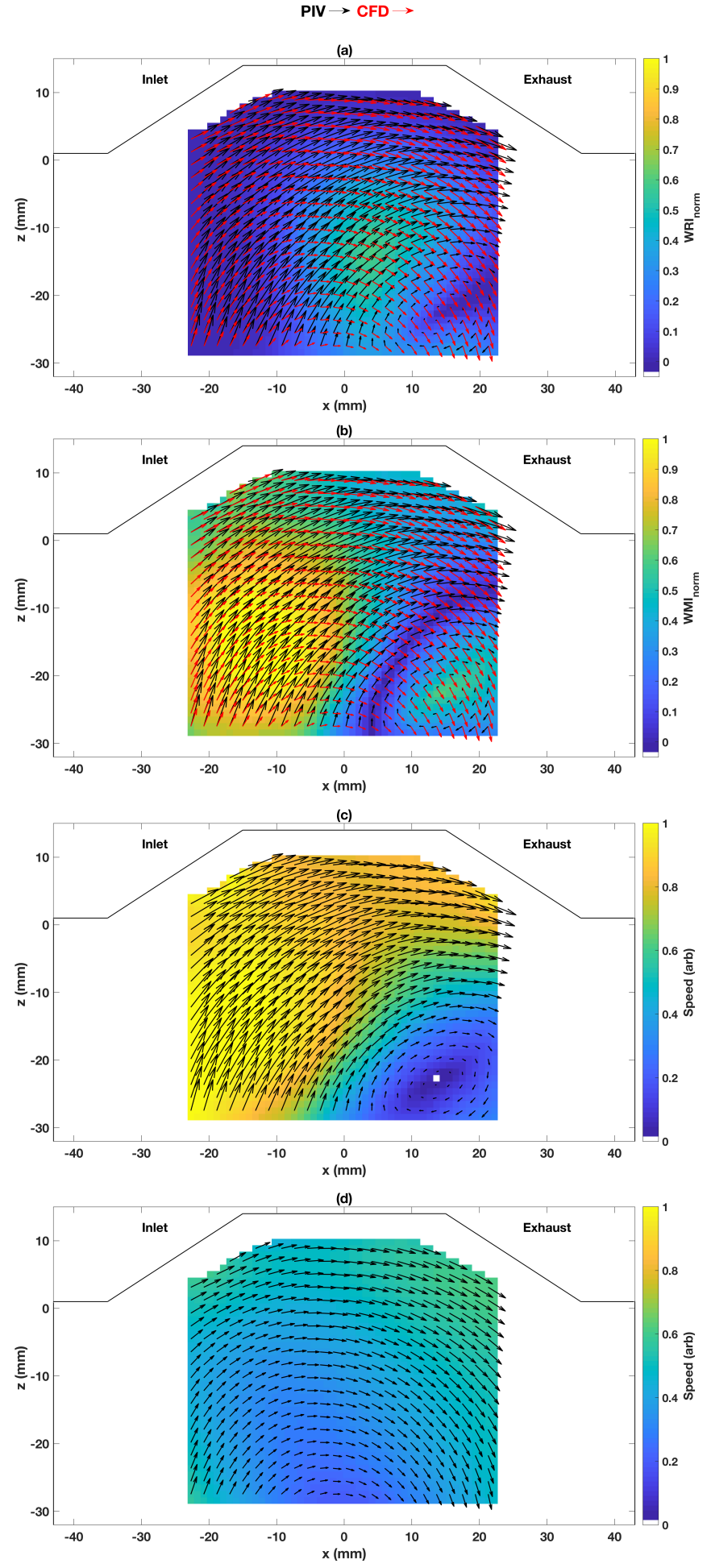

Figure 11. Plots of the WRI (a), WMI (b), and corresponding velocity fields for PIV (c) and CFD (d) for T1 at $110^{\circ} \mathrm{ca}$ bTDC (peak in the CMRI). The overlaid black and red arrows show the PIV and CFD vector fields respectively. 
A conventional method of investigating the magnitude differences between simulated and experimental flow fields is the spatially averaged flow speed. This is plotted for all conditions in Figure 12.
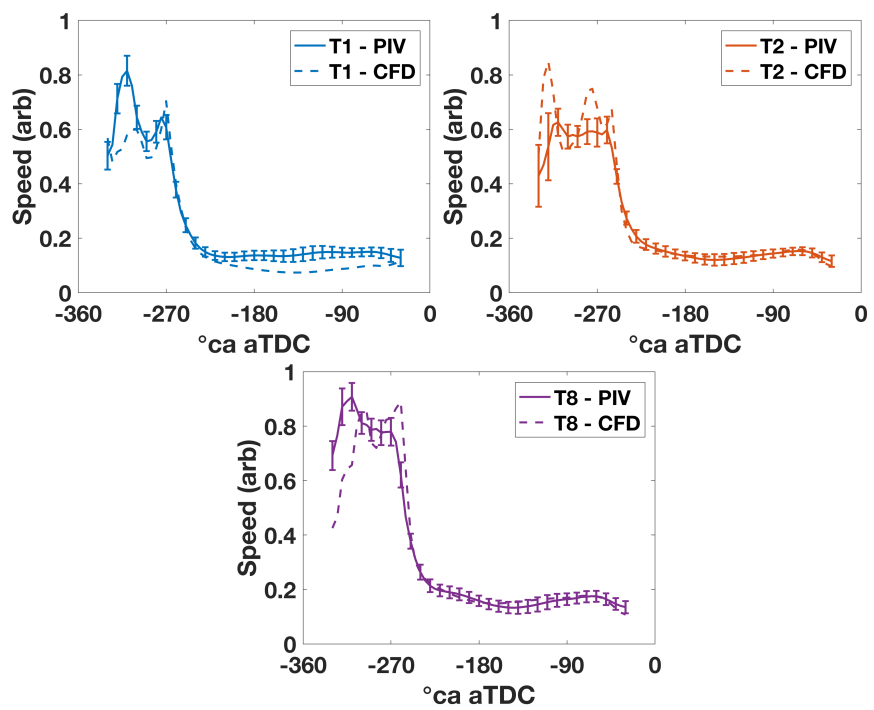

Figure 12. Plots of spatially averaged velocity magnitude for PIV experiments (solid) and CFD simulations (dashed) for T1, T2 and T8. The error bars show

$+/-1$ standard deviation for every $10^{\text {th }}$ crank angle for the PIV measurements.

It is evident that the simulated flow speeds during the compression stroke for $\mathrm{T} 2$ and $\mathrm{T} 8$ are very well matched to the experimental results. However, there is a noticeable difference for T1, where the PIV flow speed is underestimated by CFD. Although this is a very simple metric that provides a straight-forward comparison, spatial averaging can lead to potentially misleading results. For example, if one flow field has a region of slow flow on the inlet side and a region of fast flow on the exhaust side, the spatially averaged value would result in a moderate flow speed. If this is compared to a flow field that has moderate flow speed across the entire field, the resulting comparison would appear to be a good match, even though the flows have very different structures. In contrast, for the same case, the WMI would produce poor matches for both the region of slow flow and the region of fast flow, resulting in an overall poor match.

Due to the velocity weightings in the definitions of the WRI and WMI, and their normalisation to produce the CMRI, the final value that ranks the quality of the match between two velocity fields is only relative to the data that is used in the comparisons. Therefore, to put the results into perspective, Figure 13 shows equivalent plots to Figure 11, for T8, which has the lowest value of the CMRI (best match) at $110^{\circ} \mathrm{ca} \mathrm{bTDC}$.

It is clear from the velocity fields in Figure 11 (c) and (d), that there is qualitatively very good agreement between the simulated and experimental results in terms of both magnitude and alignment. This results in low spatially averaged values of the WRI and WMI, and CMRI, and demonstrates that the metrics can used to quantitatively rank comparisons of flow fields.
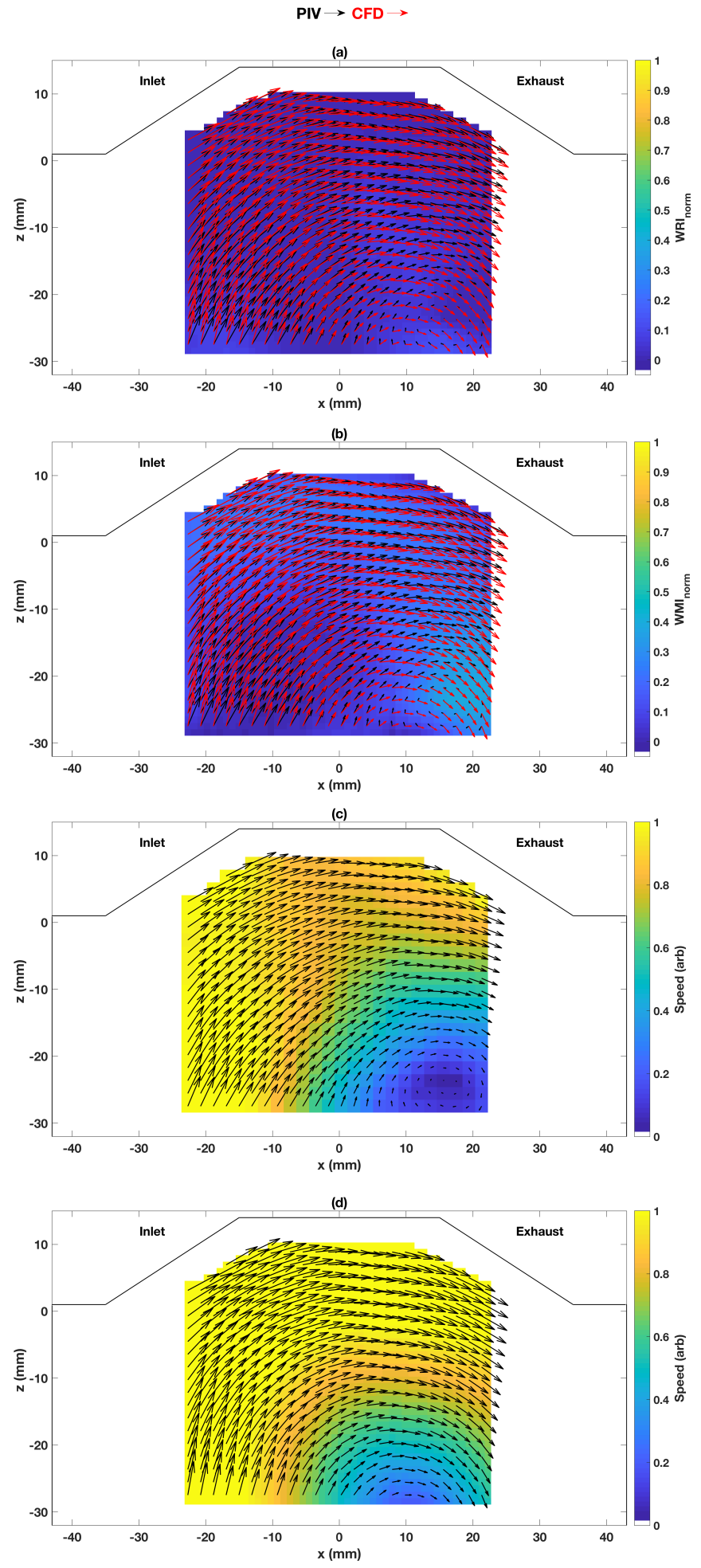

Figure 13. Plots of the WRI (a), WMI (b) and corresponding velocity fields for PIV (c) and CFD (d) for T8 at $110 \mathrm{ca}$ bTDC (lowest value of the CMRI). The overlaid black and red arrows show the PIV and CFD vector fields respectively. 


\section{Conclusions}

In this paper, novel metrics for quantifying the differences between vector fields have been used to validate RANS simulations of a GDI engine with high-speed PIV measurements. The Weighted Relevance Index (WRI) is an alignment metric that modifies the standard Relevance Index with a weighting factor that accounts for the local velocities of the two flow fields. The Weighted Magnitude Index (WMI) is a metric that weights differences in local velocities between two flow fields by an average flow speed. The WRI and WMI are then normalised and combined to produce the Combined Magnitude and Relevance Index (CMRI), which accounts for differences in alignment and magnitude with a single value.

Some researchers use a relevance index (occasionally called, more sensibly, an alignment index) to make comparisons between vector fields, but it is less usual for any comparisons to be made of the magnitudes. It can be argued that both metrics should be used, and as a first pass the CMRI can identify when there are discrepancies that can then be investigated further by separate use of the relevance and magnitude indices. Each of the MSI, RI, WRI and WMI have their uses, but if a single metric is to be used, then the CMRI has some advantages.

To demonstrate the utility of the WRI, WMI and CMRI, quantitative comparisons were made between RANS simulations and high-speed PIV measurements in an optical GDI engine for three different test conditions. Although the matches between simulation and experiment are generally very good, especially considering the lack of tuning in the underlying models, the metrics were able to identify a number of differences between the simulated and experimental results:

- An overprediction of the speed of the intake jet during the valve closing period.

- A distinct region of slow flow that appears near the intake valve directly after IVC for all test conditions is observed in the mean PIV flow fields, but not predicted by the RANS simulations.

- For a single test condition (T1), the RANS simulations underestimate the flow speeds and predict a different flow structure during the compression stroke

Although this study demonstrates the use of the WRI, WMI and CMRI for quantitative validation of CFD simulations, the metrics can be used to compare any vector fields.

It was also noted that the use of mean PIV flow fields for comparison with RANS must be treated with caution, as the inherent variation in the measured flow fields (especially in the induction process) is not considered. An interesting use of the metrics for future study would be to find the individual PIV cycle that best matches the RANS simulation and compare its performance with that of the mean PIV cycle.

\section{References}

1. G. Kalghatgi, "Is it really the end of internal combustion engines and petroleum in transport?," Applied Energy, 225:965-974, 2018, doi:10.1016/j.apenergy.2018.05.076.

2. BP Energy Economics, "BP Energy Outlook 2018,” 2018. [Online]. Available: https://www.bp.com/content/dam/bp/en/corporate/pdf/energ y-economics/energy-outlook/bp-energy-outlook-2018.pdf.

3. P. C. Miles, "History and Evolution of Optically Accessible Research and Their Impact on Our Undestanding of Engine Combustion," Proceedings of the ASME 2014 Internal Combustion Engine Division Fall Technical Conference ICEF2014-5701, 2014.

4. V. Sick, M. C. Drake, and T. D. Fansler, "High-speed imaging for direct-injection gasoline engine research and development," Experiments in Fluids, 49:937-947, 2010, doi:10.1007/s00348-010-0891-3.

5. R. Stone, Introduction to Internal Combustion Engines, 4th ed. Basingstoke: Palgrave Macmillan, 2012, ISBN:9780230576636.

6. E. Baum, B. Peterson, B. Böhm, and A. Dreizler, "On the validation of les applied to internal combustion engine flows: Part 1: Comprehensive experimental database," Flow, Turbulence and Combustion, 92(1-2):269-297, 2014, doi:10.1007/s10494-013-9468-6.

7. A. G. S. Raj, J. M. Mallikarjuna, and G. Venkitachalam, "Analysis of In-Cylinder Air Motion in a DI Diesel Engine with Four Different Piston Bowl Configuration - A CFD and PIV Comparison," SAE International Journal of Engines, 6(4):1978-1983, 2013, doi:10.4271/2013-01-2786.

8. M. M. Ameen, X. Yang, T. Kuo, and S. Som, "Using LES to simulate cycle-to-cycle variability during the gas exchange process," Proceedings of the ASME 2017 Internal Combustion Fall Technical Conference ICEF2017-3591, 2017, doi:10.1115/ICEF20173591.

9. X. Yang, S. Gupta, T. Kuo, and V. Gopalakrishnan, "RANS and LES of IC Engine Flows - A Comparative Study," Proceedings of the ASME 2013 Internal Combustion Engine Division Fall Technical Conference ICEF2013-19043, 2013, doi:10.1115/ICEF2013-19043.

10. X. Yang, S. Gupta, T.-W. Kuo, and V. Gopalakrishnan, "RANS and Large Eddy Simulation of Internal Combustion Engine Flows-A Comparative Study," Journal of Engineering for Gas Turbines and Power, 136(5):051507, 2014, doi:10.1115/1.4026165.

11. C. Pera and C. Angelberger, "Large Eddy Simulation of a Motored Single-Cylinder Engine Using System Simulation to Define Boundary Conditions: Methodology and Validation," SAE Technical Paper 2011-01-0834, 2011, doi:10.4271/2011-01-0834.

Page 11 of 14 
12.

N. Van Dam, M. Sjöberg, and S. Som, "Large-Eddy Simulations of Spray Variability Effects on Flow Variability in a Direct-Injection Spark-Ignition Engine under NonCombusting Operating Conditions," SAE Technical Paper 2018-01-0196, 2018, doi:10.4271/2018-01-0196.

13. N. Van Dam and C. Rutland, "Understanding In-Cylinder Flow Variability Using Large Eddy Simulations," Proceedings of the ASME 2015 Internal Combustion Engine Division Fall Technical Conference ICEF2015-1103, 2015, doi:10.1115/ICEF2015-1103.

14. N. Van Dam, W. Zeng, M. Sjöberg, and S. Som, "Parallel Multi-cycle LES of an Optical Pent-roof DISI Engine under Motored Operating Conditions," Proceedings of the ASME 2017 Internal Combustion Fall Technical Conference ICEF2017-3603, 2017, doi:10.1115/ICEF2017-3603.

15. K. Liu and D. C. Haworth, "Development and Assessment of POD for Analysis of Turbulent Flow in Piston Engines," SAE Technical Paper 2011-01-0830, 2011, doi:10.4271/2011-01-0830.

16. T. Wang, W. Li, M. Jia, D. Liu, W. Qin, and X. Zhang, "Large-eddy simulation of in-cylinder flow in a DISI engine with charge motion control valve: Proper orthogonal decomposition analysis and cyclic variation," Applied Thermal Engineering, 75:561-574, 2015, doi:10.1016/j.applthermaleng.2014.10.081.

17. B. Hu, S. Banerjee, K. Liu, and Q. Xue, "Large Eddy Simulation of a Turbulent Non-Reacting Spray Jet," Proceedings of the ASME 2015 Internal Combustion Engine Division ICEF2015-1033, 2015, doi:10.1115/ICEF20151033.

18. T. W. Kuo, X. Yang, V. Gopalakrishnan, and Z. Chen, "Large Eddy Simulation (LES) for IC Engine Flows," Oil and Gas Science and Technology, 69(1):61-81, 2014, doi:10.2516/ogst/2013127.

19. H. Chen, D. L. Reuss, D. L. Hung, and V. Sick, "A practical guide for using proper orthogonal decomposition in engine research," International Journal of Engine Research, 14(4):307-319, 2013, doi:10.1177/1468087412455748.

20. H. Chen, M. Xu, and D. L. S. Hung, "Analyzing In-cylinder Flow Evolution and Variations in a Spark-Ignition DirectInjection Engine Using Phase-Invariant Proper Orthogonal Decomposition Technique," SAE Technical Paper 2014-011174, 2014, doi:10.4271/2014-01-1174.

21. H. Chen, D. L. S. Hung, M. Xu, H. Zhuang, and J. Yang, "Proper orthogonal decomposition analysis of fuel spray structure variation in a spark-ignition direct-injection optical engine," Experiments in Fluids, 55:1703, 2014, doi:10.1007/s00348-014-1703-y.

22. H. Chen, D. L. S. Hung, M. Xu, and J. Zhong, "Analyzing the Cycle-To-Cycle Variations of Pulsing Spray Characteristics By Means of the Proper Orthogonal Decomposition," Atomization and Sprays, 23(7):623-641, 2013, doi:10.1615/AtomizSpr.2013007851.
23. M. M. Ameen, Y. Pei, and S. Som, "Computing Statistical Averages from Large Eddy Simulation of Spray Flames," SAE Technical Paper 2016-01-0585, 2016, doi:10.4271/2016-01-0585.

24. H. Ruhland, T. Lorenz, J. Dunstheimer, A. Breuer, and M. Khosravi, "A Study on Charge Motion Requirements for a Class-Leading GTDI Engine,” SAE Technical Paper 201724-0065, 2017, doi:10.4271/2017-24-0065.

25. M. hakariya, T. Toda, and M. Sakai, "The New Toyota Inline 4-Cylinder 2.5L Gasoline Engine," SAE Technical Paper 2017-01-0121, 2017, doi:10.4271/2017-01-1021.

26. Y. Yoshihara, K. Nakata, D. Takahashi, T. Omura, and A. Ota, "Development of High Tumble Intake-Port for High Thermal Efficiency Engines," SAE Technical Paper 201601-0692, 2016, doi:10.4271/2016-01-0692.

27. P. Abraham, D. Reuss, and V. Sick, "High-Speed Particle Image Velocimetry Study of In-Cylinder Flows with Improved Dynamic Range," SAE Technical Paper 2013-010542, 2013, doi:10.4271/2013-01-0542.

28. J. Westerweel and F. Scarano, "Universal outlier detection for PIV data," Experiments in Fluids, 39:1096-1100, 2005 , doi:10.1007/s00348-005-0016-6.

29. B. Scott, C. Willman, R. Stone, and D. Richardson, "Measurement of the Flows Induced by Fuel Injection in a GDI Engines," IMeche Engines Conference, 2018, ISBN: 978-0-9956263-4-8

30. CD-Adapco, "STAR-CD Methodolgy Manual,” 2015.

\section{Contact Information}

Blane Scott

Engineering Science, Parks Road, OX1 3PJ

michael.scott@eng.ox.ac.uk

\section{Acknowledgments}

The authors would like to thank Jaguar Land Rover Ltd for financial and technical support and thank EPSRC and Innovate UK [project 63697-464157] for partial financial support.

Page 12 of 14 


\section{Definitions/Abbreviations}

\begin{tabular}{ll} 
aTDC & After TDC \\
bTDC & Before TDC \\
CFD & Computational Fluid Dynamics \\
CMRI & $\begin{array}{l}\text { Combined Magnitude and } \\
\text { Relevance Index }\end{array}$ \\
oca & Degree crank angle \\
GDI & Gasoline Direct Injection \\
ICE & Internal Combustion Engine \\
IVC & Inlet Valve Closing \\
LES & Large Eddy Simulation \\
MSI & Magnitude Similarity Index \\
PIV & Particle Image Velocimetry \\
RANS & Reynolds Averaged Navier Stokes \\
RI & Relevance Index \\
WRI & Weighted Relevance Index \\
\hline
\end{tabular}

